\title{
Revised stellar parameters of Wolf-Rayet stars
}

\author{
Werner Schmutz and Orsola De Marco \\ Institute of Astronomy, ETH Zürich, Switzerland
}

\begin{abstract}
We discuss the reliability of the stellar parameters derived from spectroscopic analyses with atmosphere models. We follow the revisions of the stellar parameters of a WN4b star through improvements of the model codes carried out in the last ten years. In order to judge the reliability of the current results we have started to analyze binaries for which the mass of the WR star is known and therefore its luminosity can be derived from the theoretical massluminosity relation. Along this line, we present preliminary results from work in progress on the binary $\gamma^{2}$ Velorum.
\end{abstract}

\section{Introduction}

In 1990, at the IAU symposium No. 143 (van der Hucht \& Hidayat 1991), the term 'standard model' was introduced to designate the first generation of atmosphere models that could reproduce successfully the spectra of Wolf-Rayet stars (Schmutz 1991a). The basic assumptions of these models and their limitations were discussed by Hillier (1991a). Today, most of these assumptions are still necessary and even the state-of-the-art models are still based on spherically symmetric geometry, a monotonic velocity law, and time independence. However, over the past ten years there have been a number of important improvements that have allowed us to understand the dependence of the stellar parameters on some of the model assumptions. In particular, we now have a good idea of the influence of the atomic models and of the importance of the density and velocity structures on model results. Line-blanketing is nowadays included in the models and we are aware of the dependence of the ionization equilibrium of the Wolf-Rayet wind on the interaction of the He II Ly $\alpha$ transition with metal transitions at similar wavelengths.

In the next section we discuss the individual revisions of the stellar parameters by following the example of a WN4b star which has been used as a test case whenever there have been new additions to the atmosphere models. We find that the derived parameters vary considerably as a function of increasing sophistication of the models. This introduces the question of the reliability of the present results. We devote Section 3 to testing our state-of-the-art model code on the WR star in the binary $\gamma^{2}$ Vel.

\section{The dependence of the stellar parameters on model sophistication}

The most important revisions of the standard model over the past decade consist of the treatment of line-blanketing, the inclusion of metals, the addition of 
Table 1. Stellar temperatures of the WN4b star HD 50896 (WR6)

\begin{tabular}{|c|c|c|c|c|}
\hline \multicolumn{2}{|c|}{$\begin{array}{l}\text { chemical } \\
\text { composition }\end{array}$} & model characteristics & $\begin{array}{c}T_{*} \\
(\mathrm{kK})\end{array}$ & references \\
\hline $\mathrm{He}$ & & 'standard model' & 60 & H87, HSW88, SHW89 \\
\hline $\mathrm{He}$ & & +line-blanketing & 45 & S94 \\
\hline $\mathrm{He}$, & $\mathrm{N}$ & & 75 & this paper \\
\hline $\mathrm{He}$, & $\mathrm{N}$ & +el'scattering line wings & $\begin{array}{c}65 \\
100^{a}\end{array}$ & $\begin{array}{l}\text { this paper, HKW95 } \\
\text { HK98a }\end{array}$ \\
\hline $\mathrm{He}$ & & +clumping +el'sca'wings & $-b$ & H91, S95 \\
\hline $\mathrm{He}$, & $\mathrm{N}$ & +clumping +el'sca'wings & $--^{b}$ & HK98b \\
\hline $\mathrm{He}$, & $\mathrm{N}$ & +el'sca'wings +photon loss & 90 & this paper \\
\hline $\mathrm{He}$, & $\mathrm{N}$ & $\begin{array}{l}\text { +el'sca'wings +photon loss } \\
\text { +line-blanketing }\end{array}$ & - & work in progress \\
\hline $\mathrm{He}$, & $\mathrm{N}$ & $\begin{array}{l}\text { +clumping +el'sca'wings } \\
\text { +line-blanketing }\end{array}$ & 78 & C98 \\
\hline $\mathrm{He}$, & $(\mathrm{N})$ & $\begin{array}{l}\text { +clumping } \\
\text { +el'sca'wings +photon loss } \\
\text { +line-blanketing +hydrody- } \\
\text { namically calculated velocity } \\
\text { law }\end{array}$ & 84 & S97 \\
\hline
\end{tabular}

${ }^{a}$ HK98a fit the $\mathrm{N}$ spectrum only but they do not reproduce the He-line strengths.

${ }^{b}$ clumped models have been calculated, but no parameters for WR 6 have been published.

C98: Crowther (1998) based on the code of Hillier \& Miller (1998); H87: Hillier (1987a,b); H91: Hillier (1991b); HKW95: Hamann et al. (1995); HK98a: Hamann \& Koesterke (1998a); HK98b: Hamann \& Koesterke (1998b); HSW88: Hamann et al. (1988); SHW89: Schmutz et al. (1989); S94: Schmutz (1994); S95: Schmutz (1995); S97: Schmutz (1997).

electron scattering in the formal solution, the introduction of an additional free model parameter to allow for clumped winds, and the theoretical calculation of the velocity law. In order to gain a feeling for the effects that each of these revisions have on model results, we discuss them individually in the following subsections, by following the revisions of the stellar parameters of the prototype WN4b star HD 50896.

\subsection{Standard model}

There are three papers (see references in Table 1) that have analyzed HD 50896 with a standard model atmosphere, i.e., with an atmosphere composed of helium only. In Fig. 1a we show the diagnostic diagram for this star on the $T_{*}-\dot{M}$ plane. The two lines are contours of constant equivalent width for He I $\lambda 5876$ $\left(W_{\lambda}(5876)=21 \AA\right)$ and He II $\lambda 5411\left(W_{\lambda}(5411)=66 \AA ;\right.$ Hamann et al. 1993). Instead of the commonly used transformed radius for the $\mathrm{y}$-axis we give the mass loss rate for a radius of $R_{*}=2.5 \mathrm{R}_{\odot}$ and $v_{\infty}=2000 \mathrm{~km} \mathrm{~s}^{-1}$. The diagram is based on a new grid of model calculations carried out with the code of de Koter et al. (1993). The two curves indicate a solution of $T_{*} \approx 60 \mathrm{kK}$ which agrees with the results of the cited references. 


\subsection{Line-blanketing}

There are two different treatments of line-blanketing. There is the opacity sampling technique of Schmutz (1991b) with Monte Carlo simulations and there is the approach by Hillier \& Miller (1998), where the metals that are causing the blanketing are explicitly treated in the atmosphere code. The weaknesses and the strengths of these approaches are discussed in the review by Crowther (these Proceedings). Here, we would like to emphasize that the approach by Hillier \& Miller (1998) combines two different effects: the blanketing effect by tens of thousands of lines on the radiation transfer and a lowering of the degree of ionization by the presence of atoms other than helium in the model calculations. It is worthwhile to discuss separately these two effects because - as we can read in the contributions by Hillier (1999) and Crowther (1999) - the combined effect increases the inferred stellar temperatures. This seems to contradict what we know about 'ordinary' stars, for which it is common knowledge that line-blanketing lowers the deduced temperature for a given spectral type. The fact is that 'pure' line-blanketing and the introduction of metals into the model atmosphere have opposite effects but the latter is dominating in the case of WR atmospheres.

The opacity sampling technique of Schmutz (1991b) allows us to add lineblanketing to the atmosphere calculations without the burden of introducing model atoms of metals into the rate equations. Thus, atmosphere models using this method show the 'pure' effect of line-blanketing. Such calculations have been published by Schmutz (1994), who found that the deduced stellar temperatures, and thus the luminosities, are lower than those derived with the standard model. Thus, the blanketing effect on the radiation transfer, i.e., the effect of lineblanketing as it is commonly used for main sequence stars, indeed has the same effect also on the case of Wolf-Rayet atmospheres. In Table 1 we give the stellar temperature for the WN4b star HD 50896 when line-blanketed atmospheres are used. It is found that in this case the temperature is lower by $25 \%$ which implies a change of the luminosity of a factor of two.

\subsection{Metal atoms}

Line-blanketing of an atmosphere is caused by tens of thousands of lines of iron and nickel, but it is the most abundant metal that is responsible for reducing the ionization balance. In the case of a WN star the most important metal is nitrogen. Simultaneous to the inclusion of nitrogen into the atmosphere calculations, another implementation was carried out, namely the calculation of the spectral lines' electron scattering wings. In order to separate the two effects we have calculated a grid of models using the code of de Koter et al. (1993) with a nitrogen model atom with 91 levels, 1631 lines and an abundance by number of $N[\mathrm{~N}]=0.005$. In Fig. $1 \mathrm{~b}$ we show the diagnostic diagram: compared to the standard model the resulting temperature is $25 \%$ higher in the case of HD 50896.

\subsection{Frequency redistribution by electron scattering}

It was shown by Hillier (1991b) that the inclusion of the frequency redistribution by electron scattering enlarges considerably the equivalent widths of spectral lines. Since about 1993 this process is included in most of the analyses. In Fig. 1c we show the diagnostic diagram for HD 50896 based on our own calculations of 

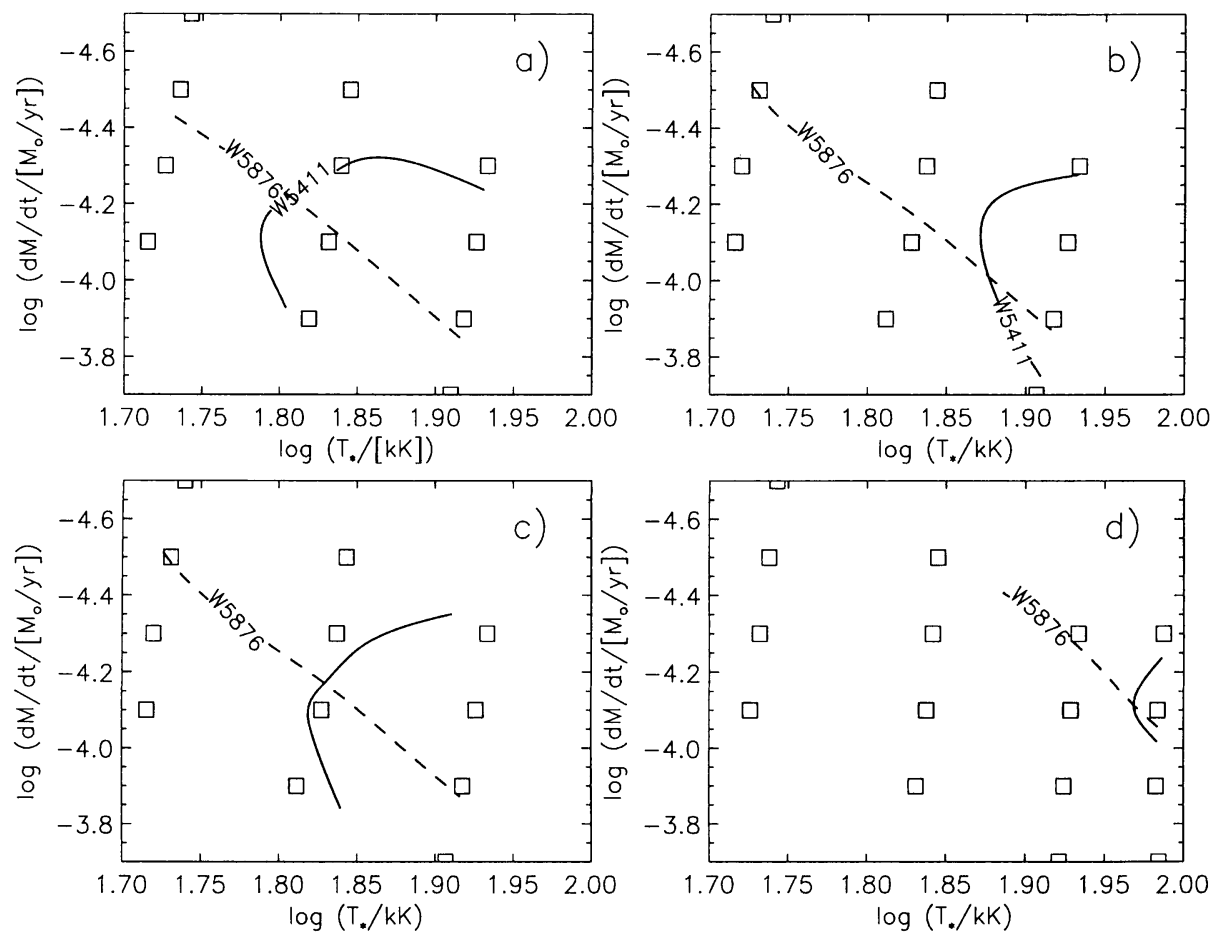

Figure 1. Diagnostic diagrams for the WN4b star HD 50896 (WR 6) based on different model predictions (see Sect.2.1.-2.7.) for the helium lines He I $\lambda 5876$ (dashed lines) and He II $\lambda 5411$ (full drawn lines). Contour lines are drawn for the observed equivalent widths (see Sect. 2.1.) on the $T_{*}-\dot{M}$ plane: (a) Standard models, i.e., He-only atmospheres; (b) He+N atmospheres; (c) as (b) but including electron scattering line wings; and (d) as (c) but including photon loss from the He II Ly $\alpha$ line.

Sect. 2.3 but now including the effect of electron scattering in the line formation. The effective temperature is $T_{*}=65 \mathrm{kK}$ which agrees well with the $T_{*}=71 \mathrm{kK}$ derived by Hamann et al. (1995).

\subsection{Clumping}

Hillier (1991b) pointed out that the electron scattering wings can be used to diagnose the clumpiness of Wolf-Rayet winds. However clumping was not included in the spectroscopic analyses until Schmutz (1994) introduced a simple approximation to implement clumping in the models. Schmutz (1997) found that the high density regions, i.e., the clumps, are a factor of four enhanced with respect to the mean density. The same technique was then used by Hamann \& Koesterke (1998b) who found similar factors for the density enhancement. There is no spectroscopic analysis of HD 50896 that would show the influence of clumping isolated from other effects but Hamann \& Koesterke (1998b) comment that clumping is of minor importance for the stellar temperature. 


\subsection{Photon loss}

Photon loss is the term used by Schmutz (1997) to describe the interaction of a strong line with metal transitions almost coincident in wavelength. This 'crosstalk' is modeled by a modification of the mean line radiation field used in the rate equations. The treatment is explained in detail in the appendix of Schmutz (1997). In principle, photon loss would be included in the treatment of line-blanketing if the lines important for the effect were taken into account correctly. However, so far the different line-blanketing treatments do not include the necessary details of line interaction so this effect has to be implemented explicitly. So far, the influence of photon loss has only been investigated on the He II Ly $\alpha$ resonance lines but there are possibly other strong metal resonance lines that are sensitive to this effect. Fig. 1d demonstrates that the photon loss through line-line interaction is an important factor in the wind ionization balance and it should not be neglected.

\subsection{Velocity law}

The biggest drawback of todays atmosphere models is that the velocity structure has to be assumed. Radiation pressure is suspected to drive Wolf-Rayet winds, but so far there is no satisfactory model that uses this mechanism to derive their velocity structure. Lucy \& Abbott (1993) have demonstrated that in principle the winds can be radiation-driven but they arrived at their result by assuming an unrealistic ionization structure which is in contradiction with observations (Schmutz 1995). Springmann (1994) and Springmann \& Puls (1998) improved the model of Lucy \& Abbott (1993), but by inspecting their diagrams (Figs. 5 and 9 in Springmann and Puls 1998), we suspect that they fail to provide the driving force in the inner part of the wind. This is where the equation of motion had its critical point and where it is determined how much mass is driven outward. Further, Springmann \& Puls (1998) do not show synthetic line profiles and therefore, their ionization structure cannot be judged against observations.

Schmutz (1997) has constructed a hydrodynamical atmosphere model for the optically thin part of the wind of HD 50896, but because of insufficient radiation force in the optically thick part, his model could not predict the inner structure. Nevertheless, the hydrodynamically calculated wind structure in the outer part brought a significant improvement of the line profile fits as demonstrated in Fig. 2 of Schmutz (1997, compare to Fig. 2 of Hamann et al. 1988). Interestingly, the calculated velocity structure differs significantly from the commonly assumed $\beta$-law with $\beta=1$. So far HD 50896 is the only Wolf-Rayet star that has been modeled with a consistent hydrodynamical density structure (albeit only for the outer part).

Comparison with the most sophisticated calculation for HD 50896 using a $\beta$-law by Crowther (1998) does not show a large difference in the stellar temperature (see Table 1). However, Crowther (1998) has neglected photon loss, so that the two models differ in two aspects. Schmutz (1997) has used a $\beta$-law with $\beta=0.5$ for the inner, optically thick part of the wind, whereas Crowther (1998) used $\beta=1$. Therefore, the comparison between the two models reflects the combined effect of the different $\beta$-laws and photon loss. We expect that the hydrodynamically calculated velocity structure of the outer part of the wind is important only for matching better the observed line profiles but that it has 
only little influence on the deduced stellar temperature. However, because of its importance for the quality of the predicted synthetic line profiles it is clear that the ability to calculate the velocity structure is of foremost importance for future improvements of the atmosphere models.

\section{Verifying the model results: the test case $\gamma^{2}$ Velorum}

The stellar temperature of HD 50896 as determined in the last ten years span the range $45 \mathrm{kK}$ to $100 \mathrm{kK}$ (Table 1 ). The corrections go in both directions but the general trend is for the temperature to increase with successive improvements of the modeling codes. The question is how far from the truth are we at present? And will future amendments to the codes change the stellar parameters further still? These questions have been with us since the first model calculations and the attempts to answer it have been many. Schmutz (1991a) has inspected the spectral predictions of the standard model and he found very good agreement with the observations. However, as Table 1 demonstrates, reproducing the observed spectrum is only a necessary requirement for the atmosphere models, but unfortunately not a sufficient one and therefore, it does not constitute a proof of the reliability of the models.

Smith \& Maeder (1989) have derived luminosities of Wolf-Rayet stars in binary systems using the theoretical mass-luminosity relation (e.g., Schaerer \& Maeder 1992). This theoretical relation has remained the same since it was introduced and it is commonly believed to be reliable. Therefore, Wolf-Rayet stars in binary systems are excellent test cases for the spectroscopic analyses because, through the masses of the components, we can derive reliably their luminosities. So far all comparisons have indicated that the spectroscopic analyses yield luminosities which are too low (see Schmutz 1996). Especially noteworthy is the case of V444 Cygni which is one of the best observed eclipsing systems with a Wolf-Rayet component. However, the distance to V444 Cygni is uncertain and we have therefore chosen $\gamma^{2}$ Velorum as a test case for which the distance was measured by Hipparcos (Schaerer et al. 1997; van der Hucht et al. 1997).

$\gamma^{2}$ Vel is a double-lined spectroscopic binary consisting of an O7.5 and a WC8 (WR11) star. A spectroscopic analysis of a binary system has to deal with the combined spectrum of two stars. As the spectra of $\mathrm{O}$ stars are well understood we have started with the analysis of the O star (De Marco \& Schmutz 1999). Except for the terminal velocity, there are no observational signatures though which to derive O star stellar wind parameters. We have therefore used the hydrodynamic atmosphere models of Schaerer \& Schmutz (1994) to infer its mass loss properties. Because the light ratio between the $\mathrm{O}$ and WR stars is subject to a very large uncertainty (compare Conti \& Smith 1972 with Brownsberger \& Conti 1993), the ratio has to be included as a parameter to be fitted in the process of the analysis. The equivalent widths measured from the observed spectrum, i.e., the combined light of the $\mathrm{O}$ and the WR star, were therefore multiplied by four different possible light ratio values. By interpolating between the predictions of 9 atmosphere models we plot in Fig. 2 contours of constant line equivalent widths in the $\mathrm{T}_{\text {eff }}-N[\mathrm{He}]$ plane. The three lines correspond to He I $\lambda 4471$, He II $\lambda 4541$ and He I $\lambda 4922$. From Fig. 2 we can see that the three contours meet in one point for a light ratio of $\Delta \mathrm{M}_{V}=1.6 \mathrm{mag}$. This yields a helium 

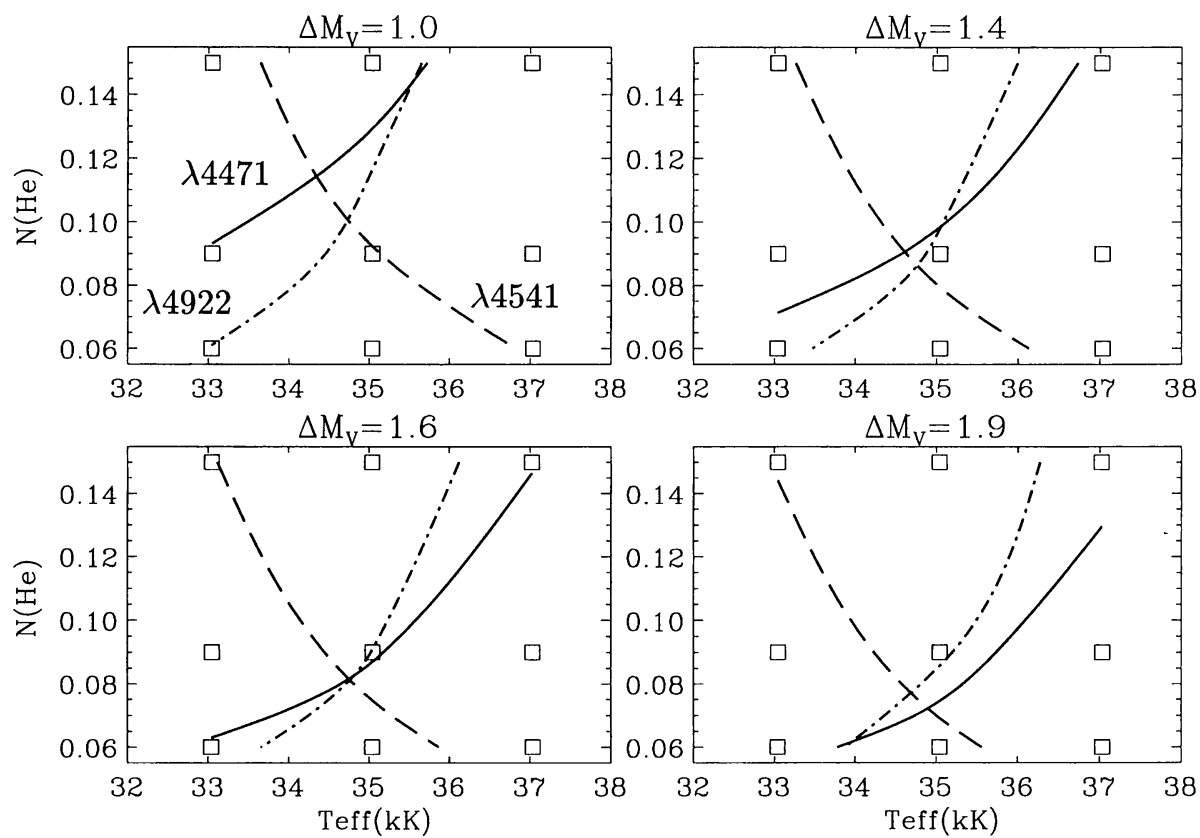

Figure 2. Diagnostic diagrams for the stellar parameters of the O- component in $\gamma^{2}$ Vel as a function of the light-ratio between the O-type star and the WR star, $\Delta \mathrm{M}_{V}$ (the O-type star is brighter). The lines denote contours for the observed equivalent widths for the He I $\lambda 4471$ (solid line), He II $\lambda 4541$ (dashed line) and He II $\lambda 4922$ (dot-dashed line). Square symbols mark the parameter coordinates for which models were calculated.

abundance that is somewhat smaller but close to the solar value. If we interpret this result to mean that helium is not enriched, then we can determine the light ratio for which the center of the triangle formed by the three contour lines lies at solar abundance. This corresponds to a light ratio of $\Delta M_{V}=1.47 \pm 0.13 \mathrm{mag}$ (where the uncertainty derives from the error bars on the line intensity measurements). We find $\log \left(L_{\mathrm{O}} / \mathrm{L}_{\odot}\right)=5.32$.

The mass of the O-type star is then determined by matching its stellar parameters with a stellar evolution track interpolated from the models of Meynet \& Maeder (1994). We find $\mathcal{M}_{\mathrm{O}}=30 \mathrm{M}_{\odot}$ and with the WR/O mass ratio of Schmutz et al. (1997), we obtain a WR mass of $\mathcal{M}_{\mathrm{WR}}=9.0 \mathrm{M}_{\odot}$. This in turn yields a luminosity of $\log \left(L_{\mathrm{WR}} / \mathrm{L}_{\odot}\right)=5.2$. Additionally, the final O star synthetic spectrum was used to deconvolve the observed spectrum of $\gamma^{2}$ Vel (see De Marco \& Schmutz 1999).

The de-convolved spectrum of the WR star was modeled by De Marco et al. (1999) with and without photon loss. We list the resulting two sets of parameters in Table 2. The effective temperature and the luminosity of the WR star is larger when photon loss is included. In Table 2 we compare the derived luminosities with that resulting from the mass-luminosity relation. The 'correct' luminosity lies between the two spectroscopic values but closer to the result for 
which photon loss is included. However, this model does not yet reflect the best possible result. Line-blanketing is still missing and from the discussion of Sect. 2.2. we expect that spectroscopic luminosity of a model with photon loss and line-blanketing will be in better agreement with the luminosity inferred from the mass-luminosity relation.

Table 2. Parameters of the WR component in the binary system $\gamma^{2}$ Vel derived from models with and without photon loss.

\begin{tabular}{lccc}
\hline & \multicolumn{2}{c}{ Model } & \multirow{1}{*}{$-L$} \\
& no photon loss & photon loss & $\begin{array}{c}\text { relation } \\
\text { ren }\end{array}$ \\
\hline$T_{*}(\mathrm{~K})$ & 75900 & 92000 & - \\
$\log \left(\dot{M} /\left(\mathrm{M}_{\odot} \mathrm{yr}^{-1}\right)\right)$ & $-4.33^{a}$ & $-4.38^{a}$ & - \\
$R_{*}\left(\mathrm{R}_{\odot}\right)$ & 1.84 & 1.78 & - \\
$\mathrm{C} / \mathrm{He}($ by number $)$ & 0.14 & 0.14 & - \\
$\log \left(L / \mathrm{L}_{\odot}\right)$ & $\mathbf{5 . 0}$ & $\mathbf{5 . 3}$ & $\mathbf{5 . 2}^{b}$ \\
& & & \\
\hline
\end{tabular}

\footnotetext{
${ }^{a}$ Homogeneous model (no clumping)

${ }^{b}$ Derived from a WR mass of $\mathcal{M}=9.0 \mathrm{M}_{\odot}$
}

\section{Discussion}

Today's state-of-the-art calculations include the following improvements over the standard Wolf-Rayet model:

- inclusion of metals, in particular of $\mathrm{N}$ for WN stars and $\mathrm{C}$ and $\mathrm{O}$ for $\mathrm{WC}$ stars;

- line formation including frequency redistribution by electron scattering;

- line-blanketing;

- line-line interaction of the He II $\lambda 303$ with metal lines either implicitly included in the line-blanketing treatment or explicitly with a photon loss formalism;

- hydrodynamically calculated velocity law of the outer, i.e., optically thin, part of the wind.

Two of these modifications, line-blanketing and electron scattering line wings lower the inferred stellar temperatures and two, inclusion of metals and photon loss, increase the stellar temperatures. The role of the hydrodynamically calculated outer velocity structure is neutral. The combined effect of the model improvements is a considerable increase of the stellar temperature compared to the results of the standard model.

In our opinion it is extremely important to verify the reliability of the atmosphere results with independent derivations of WR properties. Crowther 
(these Proceedings) has advocated the tests of the predicted emergent stellar spectrum by investigating the properties of $\mathrm{H}$ II regions around WR stars. We are actively pursuing another test: the comparison of spectroscopically derived luminosities with those inferred from the masses in WR binaries. The work in progress reported in Section 3 indicates that we are getting closer to reliable predictions of the stellar properties of Wolf-Rayet stars.

\section{References}

Brownsberger, K.R., Conti, P.S. 1993, in: J.P. Cassinelli \& E.B. Churchwell (eds.), Massive stars: their Lives in the Interstellar Medium, ASP-CS 35, 275

Conti, P.S., Smith L.F. 1972, ApJ 172, 623

Crowther, P.A. 1998, private communication

Crowther, P.A. 1999, these Proceedings

de Koter, A., Schmutz, W., Lamers, H. 1993, A\&A 277, 561

De Marco, O., Schmutz W. 1999, A\&A 345, 163

De Marco, O., Schmutz W., de Koter, A. 1999, these Proceedings

Hamann, W.-R., Schmutz, W., Wessolowski, U. 1988, A\&A 194, 190

Hamann, W.-R., Koesterke, L., Wessolowski, U. 1993, A\&A 274, 397

Hamann, W.-R., Koesterke L. 1998a, A\&A 333, 251

Hamann, W.-R., Koesterke L. 1998b, A\&A 335, 1003

Hillier, D.J. 1987a, ApJS 63, 947

Hillier, D.J. 1987b, ApJS 63, 965

Hillier, D.J. 1991a, in: K.A. van der Hucht \& B. Hidayat (eds.), Wolf-Rayet Stars and Interrelations with Other Massive Stars in Galaxies (Dordrecht: Kluwer), p. 59

Hillier, D.J. 1991b, A\&A 247, 455

Hillier, D.J. 1999, these Proceedings

Hillier, D.J., Miller D.L. 1998, ApJ 496, 407

van der Hucht, K.A., Hidayat, B. (eds.), Wolf-Rayet Stars and Interrelations with Other Massive Stars in Galaxies, Proc. IAU Symp. No. 143 (Dordrecht: Kluwer)

van der Hucht, K.A., Schrijver, H., Stenholm, B., Lundström, I., Moffat, A.F.J., Seggewiss, W., Setia Gunawan, D.Y.A., Sutantyo, W., van den Heuvel, E.P.J., De Cuyper, J.-P., Gómez, A., Marchenko, S.V. 1997, New Astronomy 2, 245

Koesterke, L., Hamann, W.-R. 1995, A\&A 299, 503

Lucy, L., Abbott, D.C. 1993, ApJ 405, 738

Meynet, G., Maeder, A., Schaller, G., Schaerer, D., Charbonnel, C. 1994, A\&AS 103, 97

Schaerer, D., Schmutz, W., Grenon, M. 1997, ApJ 484 L153

Schaerer, D., Maeder, A. 1992, A\&A 263, 129

Schmutz, W., Hamann, W.-R., Wessolowski, U. 1989, A\&A 210, 236

Schmutz, W. 1991a, in: K.A. van der Hucht \& B. Hidayat (eds.), Wolf-Rayet Stars and Interrelations with Other Massive Stars in Galaxies (Dordrecht: Kluwer), p. 39

Schmutz, W. 1991b, in: L. Crivillary, I. Hubeny \& D.G. Hummer (eds.), Stellar Atmospheres: Beyond Classical Models, NATO-ASI C341 (Dordrecht: Kluwer), p. 191

Schmutz, W. 1994, Space Sci. Rev. 66, 253 
Schmutz, W. 1995, in : K.A. van der Hucht \& P.M. Williams (eds.), Wolf-Rayet Stars: Binaries, Colliding Winds, Evolution, Proc. IAU Symp. No. 163 (Dordrecht: Kluwer), p. 127

Schmutz, W., 1996, in: J.-M. Vreux, A. Detal, D. Fraipont-Caro, E. Gosset \& G. Rauw (eds.), Wolf-Rayet Stars in the Framework of Stellar Evolution Proc. 33-rd Liège Int. Astroph. Coll., (Liège: Université de Liège), p. 553.

Schmutz, W. 1997, A\&A 321, 268

Schmutz, W., Schweickhardt, J., Stahl, O., Wolf, B., Dumm, T., Gäng, Th., Jankovics, I., Kaufer, A., Lehmann, H., Mandel, H., Peitz, J., Rivinius, Th. 1997, A\&A 328,219

Schmutz, W., 1998, in: I.D. Howarth (ed.), Boulder-Munich II: The Properties of Hot, Luminous Stars, ASP-CS 131, 119

Smith, L.F., Maeder, A. 1989, A\&A 211, 71

Springmann, U. 1994, A\&A 289, 505

Springmann, U., Puls J. 1998, in: I.D. Howarth (ed.), Boulder-Munich II: The Properties of Hot, Luminous Stars, ASP-CS 131, 286

\section{Discussion}

Langer: Firstly, its great that there are people out there (i.e., you) who believe in stellar structure predictions for the WR stars, and I think as for the $M-L$ relation you are right to do so, since this is a very firm theoretical result. But now, what about the radii. For example, Uwe Springmann told me that he gets, in his models, only sufficient force to drive the WR wind, if he adopts an inner radius for his wind which is roughly three times larger than the radius predicted by the WR stellar models. So, how do you place the inner radius of your hydrodynamic wind models?

Schmutz: The derived radii and hence, temperatures are not a reliable result of the spectroscopic analyses, at least not for those WR stars that have strong winds. The reason is that there is not yet a theory that predicts the velocity law in the optically thick part of the winds. Therefore, the derived radii simply reflect the chosen velocity law in the inner part of the atmosphere. This implies that the radius is not a good quantity to discuss. Instead it is the luminosity that should be compared with theoretical models.

Koenigsberger: Why do the models still predict P-Cygni absorption components for lines such as He I 10830 that are too strong compared with the observations?

Schmutz: This is an old problem of modeling WR spectra and it is not yet solved. I have intended already for a long time to investigate whether it could be due to re-ionization of the outer parts of the wind by X-rays. However, so far, I have never found the time to follow this idea.

Walborn: The profile of He I 10830, for which you have trouble fitting the blue-shifted absorption component, is the same as those of other subordinate (non resonance) lines in the UV, namely He II 1640 and N IV 1718. These lines all have weak absorption components relative to the emission in the P-Cygni profile. It would appear that the populations of their lower levels are smaller than in the models.

Leitherer: Could you comment on the agreement or disagreement between your massloss rate of $\gamma^{2}$ Vel and that derived from X-ray observations?

Schmutz: From what I have learned by discussing with my colleague Rolf Walder, who is an expert in hydrodynamic calculations, I understand that the results of the hydrodynamic simulations are still very uncertain. There is a lot of physics that is still not understood and the resolution of the codes do not yet allow to address their problems properly. Thus, the X-ray derived mass-loss rates are not reliable. 